\title{
GEOPOLÍTICA, DESARROLLO ECONÓMICO Y POLÍTICA EXTERIOR: JUAN ENRIQUE GUGLIALMELLI Y SU PROYECTO DE NACIÓN EN LA REVISTA ESTRATEGIA, 1969-1983
}

\author{
Geopolitics, economic development and foreign policy: Juan Enrique \\ Guglialmelli and his nation project in the Estrategia Magazine, 1969-1983
}

\author{
Cristián A. Di Renzo* \\ https://orcid.org/0000-0003-2584-6793
}

\section{Resumen}

El propósito de este artículo es analizar la producción intelectual del general de división del Ejército Argentino Juan Enrique Guglialmelli en la revista Estrategia. Concretamente, pretendemos examinar su proyecto de nación en el que se evidencia un perfil nacional-territorialista (irrendentista), en lo que respecta a la dimensión espacial, y desarrollista en lo referido al modelo económico. En los sucesivos números de la revista Estrategia elaboró una teoría pragmática acerca de cuál debería ser una adecuada conducción nacional, en materia de desarrollo económico, fronteras nacionales, Defensa, energía atómica y otras ramas centrales para la vertebración del espacio nacional. Estaba influenciado por una serie de concepciones geopolíticas que funcionan como articuladoras de todos sus trabajos.

$$
<\text { Geopolítica }><\text { Desarrollo económico }><\text { Política Exterior }><\text { Nacionalismo }>
$$

\begin{abstract}
The purpose of this article is to analyze the intellectual production of the division general of the Argentine Army Juan Enrique Guglialmelli in the Estrategia magazine. Specifically, we intend to examine his project of nation that shows a national-territorial (irrendentist) profile in regard to the spatial dimension, and developmental in relation to the economic model. In successive issues of the Estrategia magazine, he elaborated a pragmatic theory about what should be an adequate national leadership in matters of economic development, national borders, Defense, atomic energy, and other central branches for the structuring of the national space, influenced by a series of Geopolitical conceptions that function as articulators of all his works.
\end{abstract}

$<$ Geopolitics $><$ Economic development $><$ Foreign Policy $><$ Nationalism $>$

Recibido: 31/01/2020

Aceptado: 06/05/2020

\footnotetext{
* Becario Doctoral, Consejo Nacional de Investigaciones Científicas y Técnicas (CONICET), Universidad Nacional de Mar del Plata (UNDMP), Argentina, cristiandirenzo1@gmail.com
} 
Di Renzo. Geopolítica, desarrollo económico y política exterior: Juan Enrique Guglialmelli y su proyecto de nación...

\section{Introducción}

Las producciones intelectuales, ligadas a los conflictos limítrofes pendientes o acerca del aprovechamiento de los recursos naturales en zonas fronterizas - o en regiones tales como la Patagonia o el noreste argentino- son recurrentes en determinadas publicaciones periódicas en las décadas del 1960, 1970 e inicios de 1980 . Particularmente, en una reciente Tesis de Maestría (Di Renzo, 2018), evidenciamos que la Revista de Defensa Nacional, dependiente de la Escuela de Defensa Nacional, y aquellas específicas de cada fuerza siendo estas, la Revista de la Escuela Superior de Guerra (Ejército), el/la Boletín/Revista de Guerra Naval (Armada) y la Revista de la Escuela Superior de Guerra Aérea (Fuerza Aérea) presentan tales características. No obstante, nuestra investigación actual señala que tales rasgos no serían exclusivos de estas revistas específicas, sino que se extienden a otras publicaciones tales como Estrategia fundada por el general de división Juan Enrique Guglialmelli ${ }^{1}$. En esta ocasión, proponemos profundizar en el pensamiento político pragmático de su fundador y director, a través del análisis de sus artículos en el periodo 1969-1983, con el afán de contribuir a la vacancia existente dentro la historiografía abocada al estudio de la intelectualidad militar en el campo de la geopolítica.

La hipótesis que guía este trabajo es que Guglialmelli, a través de sus sucesivos trabajos, poseía un proyecto de nación en el que se evidencia un perfil nacionalterritorialista (irrendentista), en lo que respecta a la dimensión espacial, y desarrollista en lo referido al modelo económico. Asimismo, debido a la ininterrumpida utilización de concepciones geopolíticas y por el lugar que nuestro autor le otorga dentro sus trabajos, consideramos que la influencia de esta subdisciplina, de márgenes difusos dentro de las ciencias sociales, fue central ${ }^{2}$. En consonancia con esto, en un arco temporal (1969-1983) —atravesado por múltiples variables tales como la inestabilidad económica y política, con niveles de violencia inusitados-, sus trabajos mantienen una serie de elementos que permanecieron inmutables al calor de los acontecimientos, empero, atendiendo a los eventos internaciones más destacados, y con una marcada postura sobre todo en

\footnotetext{
${ }^{1}$ El presente trabajo forma parte de un proyecto de Tesis Doctoral en Historia, denominada: "De la hipótesis de guerra a la cooperación en Defensa: actores, estrategias y políticas en las relaciones entre Argentina y Chile entre las décadas de 1970-1990". De la dirección del mismo, se encuentra a cargo el Doctor Germán Soprano.

2 Preferimos la utilización de la denominación "concepciones geopolíticas" ya que, de acuerdo con lo sostenido por (Kosselleck, 1992, pp. 117), una palabra se convierte en un concepto si la totalidad de un contexto de experiencia y significado sociopolítico, en el que se usa y para el que se usa esa palabra, pasa a formar parte globalmente de esa única palabra”. A su vez, un concepto articula redes semánticas lo que le confiere un carácter inevitablemente plurívoco. Tal plurivocidad sincrónica tiene, pues, fundamentos diacrónicos (ella es un emergente de la malla de significados tejida a lo largo de su misma historia), indica una inevitable asincronía semántica. En definitiva, en un concepto se encuentran siempre sedimentados sentidos correspondientes a épocas y circunstancias de enunciación diversas, los que se ponen en juego en cada uno de sus usos efectivos (esto es, vuelve sincrónico lo diacrónico). De allí deriva la característica fundamental que distingue a un concepto: lo que lo define es, precisamente, su capacidad de trascender su contexto originario y proyectarse en el tiempo (Palti, 2007, pp. 300).

Por cuestiones de extensión y complejidad, la Antártida, Malvinas y El Atlántico Sur, serán trabajados en un artículo posterior.
} 
torno a las disputas territoriales o de aprovechamiento de los recursos naturales entre Argentina, Brasil y Chile.

Consideramos que las décadas de 1960, 1970 e inicios de 1980, son de fuerte presencia de la corriente nacional-territorialista (Cavaleri, 2004; Di Renzo, 2018; Escudé 2008) que utilizan una amplia gama de concepciones geopolíticas en sus producciones intelectuales. Figuras de renombre dentro de las Fuerzas Armadas — tales como la del general de división Osiris Villegas o la del almirante Isaac Rojas- publicaban en revistas, libros, conferencias y cursos especiales, acerca de la importancia de la conservación del territorio nacional y del aprovechamiento de los recursos en áreas fronterizas. Pues, precisamente, en este periodo tienen lugar el Conflicto Beagle entre Argentina y Chile $y$, por otro lado, las diferencias existentes por la explotación de los recursos hídricos de la Cuenca del Plata entre Brasil y Argentina. En una reciente investigación (Di Renzo, en prensa), planteamos la hipótesis de que la geopolítica ofreció, desde sus diferentes vertientes interpretativas, una amplia gama de concepciones que permearon durante el recorte temporal seleccionado, a una gran parte de las producciones intelectuales argentinas ligadas al análisis de los conflictos limítrofes pendientes, al aprovechamiento de los recursos en las zonas fronterizas y a otros temas afines. Tales concepciones giran en torno al aparente expansionismo territorial de los países vecinos, la necesidad de afianzar el Estado en zonas fronterizas, la historia (equívoca) de la política exterior, el factor poblacional, la relación entre extensión del territorio y fortaleza de la nación, la proyección y posicionamiento en el esquema sudamericano por parte de Argentina en calidad de potencia-, entre otras ${ }^{3}$. Estas ideas, que fueron planteadas hacia finales del siglo XIX e inicios del siglo XX, son retomadas por diversos intelectuales civiles y militares en el periodo seleccionado, dando como resultado la existencia de una corriente nacional-territorialista (irrendentista) de la cual consideramos que el general de división Guglialmelli formaba parte, ya que su proyecto de desarrollo económico fue planteado en tales términos ${ }^{4}$.

Para dar cuenta de estos aspectos, en primer lugar, nos detendremos en la figura de Juan Enrique Guglialmelli y cuestiones generales de la revista Estrategia, para posteriormente, trabajar sobre sus artículos (38 en total, uno de ellos publicado post-mortem en el año 1983 y el número especial dedicado a la Guerra de Malvinas)

3 En este sentido, nuestro trabajo se encuentra en sintonía con aquellos que consideran que la geopolítica servía de caja de herramientas a líderes (militares) que pensaban en sus términos, proveyendo explicaciones sobre un adecuado desarrollo nacional, integración territorial y sobre las relaciones con los países vecinos (Fornillo, 2015). A su vez, coincidimos con (Deciancio, 2017, p. 200), que sostiene que "durante la última dictadura civil-militar en Argentina, la geopolítica se convirtió en una caja de herramientas que proveyó una explicación consistente sobre los esquemas de desarrollo nacional, integración territorial, las relaciones con sus vecinos y el mundo".

4 El nacionalismo territorial es un fenómeno contemporáneo de gran interés intelectual y relevancia política variable. Lo que lo hace intelectualmente interesante es el contraste entre la percepción de pérdidas territoriales y la realidad objetiva de ganancias territoriales. Se diferencia del nacionalismo económico y político argentino en tanto los grupos políticos, que propician estas diversas variantes de nacionalismo, no siempre coinciden. La combinación de nacionalismo territorial con liberalismo económico, por ejemplo, han sido frecuente (Escudé 2008, p. 2). Acerca de la existencia de una corriente nacional-territorialista (irrendentista), véase Di Renzo (2018). 
Di Renzo. Geopolítica, desarrollo económico y política exterior: Juan Enrique Guglialmelli y su proyecto de nación...

que tuvieron aparición entre los años 1969 y $1983^{5}$. Este trabajo presenta un esquema dividido en cuatro secciones: la visión geopolítica del espacio nacional, la visión geopolítica sobre el Brasil, el desarrollo nacional y el rol de las Fuerzas Armadas, y el conflicto Beagle. Para finalizar, realizaremos algunas reflexiones generales, a modo de propuesta, para futuras líneas de investigación y debate en torno a la influencia de la geopolítica en las producciones intelectuales en el periodo seleccionado.

\section{Juan Enrique Guglialmelli y la Revista Estrategia}

Juan Enrique Guglialmelli nació el 22 de diciembre de 1917, en la localidad de San Martín, provincia de Buenos Aires. Proveniente de una familia de militares, egresa del Colegio Militar como subteniente de comunicaciones en el año 1938. Once años más tarde, ingresa a la Escuela Superior de Guerra para obtener el título de Oficial de Estado Mayor. En el año 1952, es declarado en situación de retiro obligatorio. Habría sido el general Juan Domingo Perón quien influye en esa medida, porque consideraba al joven oficial tan inteligente como peligroso (Larra, 1995, p. 15), retirándose a la ciudad de Goya en donde se desempeñó como carnicero. Tras el Golpe de Estado de 1955, es reincorporado a la actividad, ascendido al grado de mayor y con destino en la secretaría del general Arturo Ossorio Arana. Posteriormente, es trasladado como jefe de la IV División del Ministerio del Ejército, por haber convenido una cita con Arturo Frondizi, quien ya aparecía como candidato presidencial. Esta situación habría alterado al general Aramburu, y el nuevo destino respondería a una estrategia para alejar a Guglialmelli (Larra, 1995, p.17). El 16 de octubre de 1959, es designado como Secretario de Enlace y Coordinación de la Presidencia de la Nación, aunque días después es nombrado como Asesor Delegado Militar argentino ante la Junta Interamericana de Defensa, cargo en el que se desempeñará hasta finales del año 1961. En febrero de 1962, es nombrado jefe del Estado Mayor en el Comando del IV Cuerpo de Ejército y en octubre recibe la Comandancia de la División de Infantería de Montaña (Neuquén). El 15 de enero de 1965, es ascendido a general de brigada y en junio del mismo año asume la Dirección de la Escuela Superior de Guerra y del Centro de Altos Estudios.

Con la llegada al poder mediante un Golpe de Estado, en el año 1966, la dictadura civil-militar — autodenominada Revolución Argentina- se prometía transformar la realidad socio-económica del país, con algunos planteos que, al menos a nivel conceptual, se acercaban a las propuestas de Guglialmelli 6 . Durante este período, en cuanto a su carrera militar, se desempeña como comandante del V Cuerpo del Ejército (Bahía Blanca) —diciembre de 1966- y el 10 de octubre de 1967 se lo promueve a

\footnotetext{
5 La mayor parte del corpus documental pertenecen al archivo del investigador. Los números faltantes de la colección fueron relevados a partir de la compilación realizada por la Universidad Nacional de Lanús (2007) en el que se transcribieron los artículos del general Guglialmelli.

6 La dictadura autodenominada Revolución Argentina comprendió tres periodos presidenciales: Juan Carlos Onganía (junio de 1966-junio de 1970), la del general Marcelo Levingston (junio de 1970-marzo de 1971) y la del general Alejandro Agustín Lanusse (marzo de 1971-mayo de 1973). Culmina con la asunción a la presidencia, tras elecciones, de Héctor Cámpora.Acerca del Golpe de Estado, y del funcionamiento del gobierno de facto de la Argentina, véase O'Donnell (1981).
} 
general de división. Finalmente, se lo declara en situación de retiro el 7 de noviembre de 1968.Un dato a destacar es que durante la presidencia, de facto, de Marcelo Levingston, se desempeñó como secretario de la Comisión Nacional de Desarrollo (CONADE), uno de los dos organismos específicos, junto con el Consejo Nacional de Seguridad (CONASE), destinados a la implementación de la Doctrina de Seguridad Nacional y Desarrollo en la dictadura civil-militar de la Revolución Argentina. ${ }^{7}$ No obstante, su desempeño en este cargo no se extendió por mucho tiempo (entre el 30 de junio y el 3 de noviembre de 1970), ya que Guglialmelli decide renunciar al mismo, por motivos que declara en su obra 120 días en el gobierno ${ }^{8}$. Desde el momento en que se conoció su renuncia, se desató una campaña tendiente a desvirtuar su postura y posicionarlo como un acto solidario con el paro propiciado por la CGT (Larra, 1995, p. 29).

En abril de 1971, cumple con el arresto de 60 días dictado por Alejandro Lanusse en Diamante, provincia de Entre Ríos. Los motivos tendrían relación con una nota publicada en el Diario Clarín, en donde Guglialmelli esgrime argumentos en contra del Gran Acuerdo Nacional y realiza críticas contra el gobierno de facto. No obstante, como veremos, algunas de ellas tienen lugar en la revista Estrategia, fundada por él mismo en el año 1969. De aparición bimestral, Estrategia era el órgano de difusión principal del Instituto Argentino de Estudios Estratégicos y de Relaciones Internacionales (INSAR). Dentro de los intelectuales que contribuyen en sus números, encontramos tanto a civiles como militares ${ }^{9}$. En lo que respecta al público lector, al que se dirigían los escritos, podemos inferir, por el uso de conceptos especializados, por la participación de figuras destacadas de cada rama interviniente y por las problemáticas abordadas, de que se trataba de pares académicos, intelectuales militares o políticos con el perfil propio de los artículos ${ }^{10}$. En otras palabras, el público en general podía no tener

\footnotetext{
Al respecto, véase Duca (2010), Leal Buitrago (2003) y Rivera (2002).

8 Durante su gestión como secretario del CONADE, a partir del 30 de junio de 1970, Guglialmelli elaboró el "Plan CONADE" y se enfrentó abiertamente al plan de estabilización del ministro de Economía Moyano Llerena. Guglialmelli era partidario de una política industrialista de signo antiliberal: aumentos masivos de salarios, desgravaciones impositivas, y protección a la empresa argentina. Asimismo, la sugerencia de Guglialmelli de nacionalizar los depósitos bancarios lo enfrentó tanto con el "liberal" Moyano Llerena como con el "desarrollista" Aldo Ferrer. El 3 de noviembre de 1970, renunció a su cargo tras declinar el ofrecimiento que le hiciera Levingston de ser ministro del Interior. En el texto de su renuncia, Guglialmelli denunciaba que monopolios internacionales estaban interfiriendo en las decisiones del Estado argentino. Al respecto véase Cisneros y Escudé (2000).

9 Debemos destacar contribuciones de personalidades del ambiente público y académico, tales como ex ministro de defensa Oscar Camillón, los historiadores Bruno Passarelli y Ricardo Callet Bois, el ensayista Félix Luna, el político peronista Alberto Assef, entre otros. Dentro del universo militar, se destacan las participaciones del general de división (RE) Eduardo Juan Uriburu, el general Osiris Villegas, el coronel Benjamín Rattenbach y el coronel (RE) Florentino Díaz Loza. Un lugar aparte merecen las reproducciones de los textos del general del Ejército Francés André Baufré, con numerosos artículos destinados a su persona y sobre su pensamiento estratégico militar.

${ }^{10}$ Para el análisis de la fuente seleccionada, nos serviremos de las herramientas teóricas provenientes del campo de la Historia Intelectual, especialmente aquellas en las que se prioriza las formas en las que los pensamientos se insertan y se reproducen en un determinado tiempo y espacio (Di Pasquale, 2011). Asimismo, utilizaremos la denominación de conceptos o concepciones ya que, en consonancia con Kosselleck (1992, p. 117), una palabra se convierte en un concepto si la totalidad de un contexto de
} 
Di Renzo. Geopolítica, desarrollo económico y política exterior: Juan Enrique Guglialmelli y su proyecto de nación...

acceso a estos contenidos especializados, en donde se producían análisis que requerían cierta formación intelectual o académica ${ }^{11}$.

En cuanto a los objetivos de la publicación, los mismos giran en torno al análisis de diversos núcleos temáticos, con la finalidad de darlos a conocer, de encontrar soluciones prácticas y de generar una "conciencia nacional". En este sentido, no podemos dejar de lado que la sintaxis de las revistas lleva las marcas de la coyuntura en la que su actual pasado era presente (Sarlo, 1992, p. 10) y que, además, su accionar, su intervención en tal contexto, apunta a transformarlo. En el caso de la fuente seleccionada, esto se manifiesta número tras número, bajo el lema de "libertad académica", empero sin renunciar a una línea propia que respondería a la necesidad de cambio que demandaba los siguientes puntos:

"-Objetivos nacionales y políticos claros y definidos.

-Política exterior independiente capaz de obtener la libertad de acción necesaria para el logro de esos objetivos.

-Colaboración de los distintos sectores de nuestra sociedad y su participación efectiva en el QUE y COMO hacer concretos. -Cabal conocimiento de los intereses externos e internos en conflicto con los propios fines perseguidos, sus modos de operar y sus agentes.

-Un programa de desarrollo económico, social y cultural ejecutado con ritmo acelerado y definidas prioridades, en áreas geográficas rezagadas, sectores básicos de la producción industrial e infraestructura de servicios, en el diálogo social y en una política educacional y de investigación científica y tecnológica al servicio del desarrollo nacional"12.

Concretamente, desde las primeras páginas de este órgano de difusión, Guglialmelli deja en claro cuál debe ser la línea editorial para aquellos escritores, militares o civiles que contribuirán a lo largo de su aparición. Sobre este punto, volveremos más adelante. En cuanto al régimen de publicación de la revista, la misma era de carácter bimestral (en ocasiones aparecieron dos números juntos) y tuvo existencia entre los

experiencia y significado sociopolítico, en el que se usa y para el que se usa esa palabra, pasa a formar parte globalmente de esa única palabra. También coincidimos en que la característica fundamental que distingue a un concepto es, precisamente, su capacidad de trascender su contexto originario y proyectarse en el tiempo (Palti, 2007, p. 300).

${ }^{11}$ El trabajo con un corpus documental de estas características presenta una serie de elementos que le son propios. Siguiendo la línea argumental de Beatriz Sarlo, consideramos que las revistas brindan información sobre las costumbres intelectuales de un periodo y grupo social específico, sobre las relaciones de fuerza, poder y prestigio en el campo de la cultura, relaciones y costumbres que no repiten de manera simple las que pueden leerse en los libros editados contemporáneamente (Sarlo, 1992, p. 15). Por lo tanto, la Revista Estrategia, nos permite obtener además, un abordaje de su contemporaneidad privilegiado, siendo expresión de diversos momentos de crisis, sobre todo, ligadas a la política exterior argentina, latinoamericana y en escenarios de conflicto dentro del mundo bipolar, que a su vez responden al enfoque analítico de la misma.

${ }^{12}$ Guglialmelli, J., "Propósitos y definiciones”. Estrategia n¹, mayo-junio, 1969, p. 7. 
años 1969 y $1983^{13}$. Pasemos entonces, al análisis correspondiente al razonamiento geopolítico (Mendoza Pinto, 2017) que Guglialmelli tiene sobre el espacio nacional.

\section{Visión geopolítica del espacio nacional}

Este aparatado se encuentra destinado al análisis, que tenía este general del Ejército Argentino, de las concepciones geopolíticas acerca del espacio nacional. Comenzaremos señalando que desde mediados del siglo XX, en América Latina, crece el campo de los estudios geopolíticos. Tal como señala Quintero (2004), los militares de Argentina, Brasil y Chile apostaron por el desarrollo de los centros dedicados al estudio de este campo en las revistas especializadas y las cátedras en institutos de formación militar y diplomática. No obstante, añadiremos que si bien se realizan "denuncias" en las producciones intelectuales de militares argentinos, frente al expansionismo vecinal en las fronteras, no existían homogeneidades en torno a una aparente postura belicosa asociada a tales concepciones. Pues, como veremos, nuestro autor apuesta a un modelo de desarrollo económico integral más que a una salida armada de los conflictos territoriales.

Si bien no es el objetivo central de este trabajo describir de cuál o cuáles ramas de la geopolítica se desprenden las concepciones de Guglialmelli, señalaremos que toma elementos tanto de la Geopolítica Clásica como la Posclásica, para adaptarlos al contexto argentino en el cual escribe ${ }^{14}$. Asimismo, dentro de este campo en Argentina, y en particular dentro del Ejército, podemos situarlo dentro de la línea de Jorge Atencio y de Felipe Marini ${ }^{15}$.En lo que respecta a los referentes del Ejército citados por Guglialmelli para sus trabajos, encontramos al teniente general Pablo Ricchieri, al general de brigada Alonso Baldrich y al general de división Enrique Mosconi, todos ellos ligados a sectores claves para el desarrollo económico, tales como la energía, la minería, la siderurgia y la química pesada. Además de estos referentes, resalta la figura del general de división Manuel Savio, uno de los íconos de la proyección militar-industrial en Argentina (Avellaneda, 2019), al que cita en reiteradas ocasiones, sobre todo por su célebre frase "la presión económica ciñe más fuerte que la presión bélica: no es cruenta, pero es implacable e integral" "16. Por lo tanto, la generación militar de las primeras décadas

\footnotetext{
${ }^{13}$ A su vez, el arco temporal, nos impone el desafío de contextualizar cada periodo histórico, empero, sólo realizaremos algunas referencias generales para intentar no desviarnos de los objetivos propuestos para este trabajo.

${ }^{14}$ Utilizamos el modelo propuesto por Agnew (2005), quien señala que la geopolítica atravesó por tres grandes etapas: la Clásica o tradicional, compuesta por los inicios de la disciplina, en la que se diferencian tres vertientes (civilizadora, naturalista e ideológica); la posclásica, que se desarrolla durante la Guerra Fría, contexto en el que situamos los trabajos de Guglialmelli; y posmoderna o crítica para hacer referencia a los teóricos post caída del Muro de Berlín.

${ }^{15}$ Ambos integrantes del Ejército Argentino se destacaron por sus contribuciones al campo de la geopolítica. En el caso de Jorge Atencio, su obra de mayor repercusión es ¿Qué es la geopolítica?, del año 1965, mientras que en el caso de Felipe Marini se destaca El conocimiento Geopolítico de año 1985. Al respecto véase Mendoza Pinto (2007), y sobre la perspectiva geopolítica de Marini, véase Fernandes (2013).

${ }^{16}$ Los artículos son Guglialmelli, J. "Fuerzas Armadas para la liberación nacional". Estrategia n²3, julioagosto de 1973, pp. 144, en Guglialmelli, J. (2007); “Argentina- Brasil, enfrentamiento o alianza para la liberación”. Estrategia n ${ }^{\circ}$ 36, septiembre-octubre de 1975, p. 19; "Argentina, Política nacional y problemas
} 
Di Renzo. Geopolítica, desarrollo económico y política exterior: Juan Enrique Guglialmelli y su proyecto de nación...

del siglo XX enarboló las banderas de la industrialización, a instancias de alcanzar la autonomía en la producción de materiales bélicos y así sostener el esfuerzo de una hipotética guerra (Cornut, 2018, p. 168). En este sentido, el desarrollo económico actuaría, a su vez, como mecanismo de defensa sobre cualquier tipo de amenaza, sobre todo en las fronteras con los países limítrofes de Brasil y Chile.

En principio, es interesante destacar que su concepto de frontera se encuentra alejado de la consideración de que es un mero límite, diferenciando ambos conceptos, ya que entiende que:

"La frontera es el ámbito geográfico que, a partir de dicho límite, se extiende hacia el interior del propio territorio hasta una distancia determinada. Como este espacio está en contacto con el país vecino, es lícito afirmar que frontera es el ámbito geográfico de dos naciones, a ambos lados del linde. Éste, en síntesis, es un concepto lineal. La frontera una noción geoambiental" ${ }^{17}$.

Así, al considerar la frontera desde esta concepción, plantea la necesidad de entenderla a través de múltiples aspectos, tales como la situación socio-económica de cada país en general y en el espacio fronterizo propiamente dicho. Pues, como veremos, el desarrollo integral de la nación sería un factor clave para frenar los supuestos avances expansionistas de los países vecinos de Brasil y Chile. Por consiguiente, no bastaría simplemente con militarizar o custodiar las fronteras para impedir tales avances, sino que se plantea la necesidad de implementar un proyecto de nación integral. En consonancia con esto último, reconoce que el problema en lo que considera como fronteras periféricas (zonas alejadas de la Capital, tales como la Patagonia y el Noreste) radica en una variable histórica de larga data: el eje portuario Buenos Aires-Rosario fomentado por el proyecto de nación de la Generación del '80. Partiendo de un diagnóstico de crisis, sostiene que la nación le dio la espalda a las regiones de la Mesopotamia, el espacio marítimo, el Atlántico Sur, la Patagonia y la Antártida y por ello se encuentra vulnerable frente al expansionismo de Brasil, Chile y Gran Bretaña. Particularmente, acerca de la Patagonia, zona considerada como de mayor vulnerabilidad, nuestro autor sostiene que la solución se encontraría en la promoción de polos de desarrollo de las distintas zonas, en función de las posibilidades energéticas que pueda ofrecer. Además, en la creación de infraestructura destinada a su integración, tales como puertos, aeródromos, ferrocarriles y caminos ${ }^{18}$.

de fronteras. Crisis nacional y problemas fronterizos". Estrategia n $^{\circ} 37 / 38$, noviembre-diciembre de 1975 y enero y febrero de 1976, pp. 10; ¿Argentina insular o peninsular? Reflexiones geopolíticas en torno al pensamiento del Vicealmirante Segundo R. Storni". Estrategia ${ }^{\circ}$ 40/41, mayo- junio, julio-agosto de 1976, p. 25; "Economía, Poder Militar y Seguridad Nacional". Estrategia n 51, marzo-abril de 1978, pp. 8 y 19; “Argentina. Geopolítica y fronteras. Estrategia n ${ }^{\circ}$ 57, marzo-abril de 1979, pp. 6 y 13; y el artículo especialmente dedicado a su persona, denominado "El General Savio. Industrias básicas, poder militar y poder nacional". Estrategia n60, septiembre-octubre de 1979, pp.5-36.

${ }^{17}$ Guglialmelli, J., "Argentina, Política nacional y problemas de fronteras. Crisis nacional y problemas fronterizos". Estrategia n³7/38, noviembre-diciembre de 1975 y enero y febrero de 1976, pp. 5.

${ }^{18}$ Guglialmelli, J. "Los intereses vinculados al statu quo postergan la integración patagónica", Estrategia, 
Figura 1. Esquema de los polos de desarrollo ${ }^{19}$

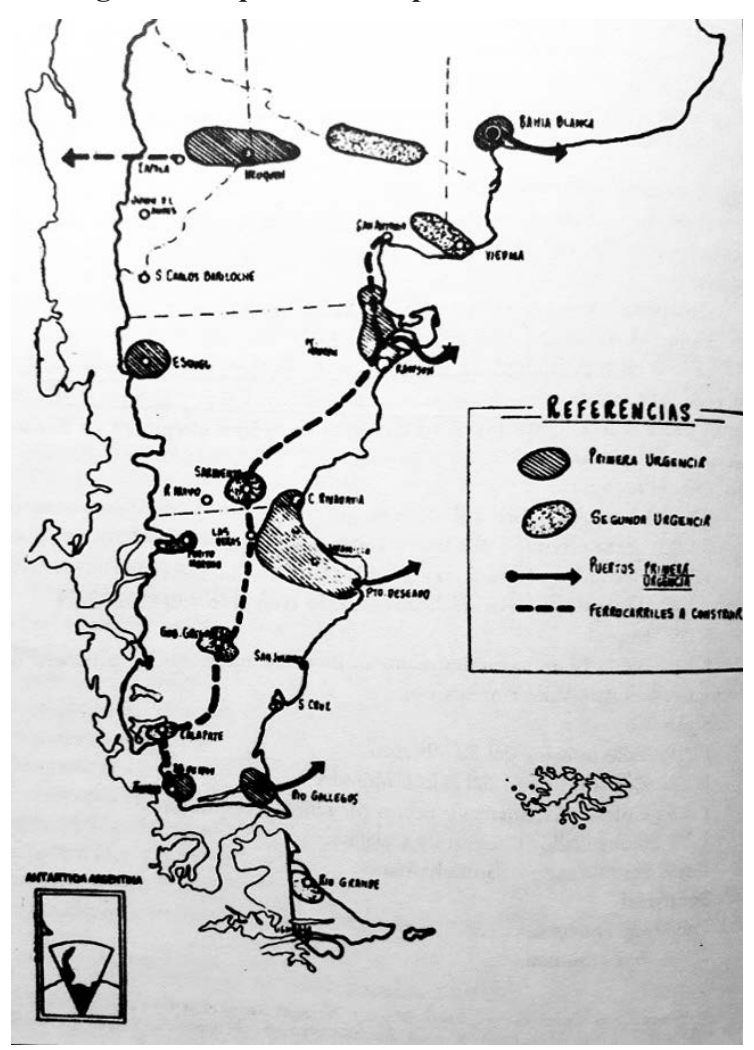

En la imagen se detalla los sitios que requieren una actuación inmediata, señalada como de primera urgencia y otros de segunda urgencia, pero no por ello menos importantes. Estos constituirían los polos de desarrollo, destinados a la vertebración del espacio nacional en la región patagónica, sitio en el cual Guglialmelli se desempeñó durante su carrera militar.

Como podemos evidenciar su concepción de frontera se encuentran interrelacionadas al aparente expansionismo territorial de los países vecinos, la necesidad de afianzar el Estado en zonas fronterizas y en la correspondencia entre extensión del territorio y fortaleza de la nación. Argumentos utilizados por parte de la corriente nacionalterritorialista (irrendentista). La noción de riego es entendida en términos geopolíticos, y se encontraría representada por los países vecinos pero también por Gran Bretaña en su accionar en las Islas Malvinas y sus pretensiones en la Antártida. Los vacíos demográficos - y de escasa presencia estatal en las áreas que considera como vulnerables-, se

n³, septiembre-octubre de 1969, pp. 8-23; Guglialmelli, J. "Patagonia. A cien años de su ocupación no podemos conmemorar su vertebración a la nación”, Estrategia, n59, julio-agosto de 1979, pp. 5-36.

${ }^{19}$ Guglialmelli, J. "Los intereses vinculados al statu quo postergan la integración patagónica”, Estrategia, n³, septiembre-octubre de 1969, p. 20. 
Di Renzo. Geopolítica, desarrollo económico y política exterior: Juan Enrique Guglialmelli y su proyecto de nación...

convierten en uno de los objetivos geopolíticos de su modelo de desarrollo nacional. Por ende, la Cuenca del Plata, el Canal Beagle, el espacio antártico, Malvinas y el Atlántico Sur son los objetivos geopolíticos de desarrollo del modelo pragmático de Guglialmelli ${ }^{20}$. En términos de códigos y siguiendo los lineamientos de Flint (2006), nuestro autor delimita quiénes son los actuales y potenciales enemigos y cómo enfrentarlos de manera eficiente. Pasemos entonces al análisis de uno de ellos (tal vez el más importante), Brasil, en una de las zonas que considera de riesgo: la Cuenca del Plata.

\section{Visión geopolítica sobre Brasil: denuncias sobre expansionismo y proyectos incompatibles con el desarrollo nacional}

Las relaciones entre Argentina y Brasil ocupan una buena parte de los trabajos de Guglialmelli en la revista Estrategia. Particularmente, la problemática asociada a la relación entre ambos países en el periodo citado es la explotación de los recursos hídricos en la Cuenca del Plata ${ }^{21}$. En este sentido, nuestro autor no se hallaba exento del clima reinante entre ambos países y su producción intelectual da cuenta de ello.

En el año 1973, teniendo como antecedente el Tratado de Yguazú (1966), Brasil y Paraguay firman el Tratado de Itaipú mediante el cual se acuerda la construcción de una represa hidroeléctrica sobre el río Paraná, la cual sería emplazada a $17 \mathrm{~km}$ de la frontera con Argentina. Coincidimos con los planteos que aseguran que la llegada del nuevo gobierno de Cámpora no implicó un cambio en la perspectiva geopolítica argentina (Cisneros y Escudé, 2000), recelosa de lo efectuado por las autoridades brasileñas en materia energética en la Cuenca del Plata. Posición que podemos extender, con matices, a las presidencias de Lastiri, Perón y Martínez de Perón. La estrategia de Juan Domingo Perón parece haber sido reservar la cuestión del principio de "consulta previa", para ponerla a consideración de la Asamblea General de la ONU, que tendría lugar a fines de noviembre. De este modo, el 27 de noviembre de 1973, la delegación argentina logró imponer el principio de consulta previa en la segunda comisión de la Asamblea General de la ONU, por un resultado de 78 votos a favor, 6 en contra (Bolivia, Brasil, Francia, Nicaragua, Paraguay y Portugal) y 41 abstenciones. El proyecto de resolución argentino, titulado "Cooperación en el Campo del Medio Ambiente en materia de Recursos Naturales compartidos por dos o más Estados", contó con el patrocinio de 52 países, y reiteraba el principio de consulta previa sustentado por Cuba y la Argentina en la Conferencia de No Alineados en Argel, donde había sido respaldado por 76 países. Esto produjo un clima conflictivo entre Argentina y Brasil, expresado en la Sexta (entre fines de mayo e inicios de junio de 1975, en la ciudad de Cochabamba, Bolivia) y la Séptima Reunión de Cancilleres de la Cuenca del Plata (en junio de 1974, en la ciudad Buenos Aires, Argentina). Habrá que esperar hasta que el gobierno de facto del Proceso

${ }^{20}$ Por cuestiones de extensión y complejidad, la Antártida, Malvinas y el Atlántico Sur, serán trabajados en un artículo posterior.

${ }^{21}$ El conflicto tiene inicio con la firma del Acta de Yguazú, entre Brasil y Paraguay, del año 1966. En el mismo se plantea el proyecto de construir una usina binacional. Al respecto véase Fajardo, J (2004) y Segre (1990). 
retome la senda de las negociaciones, ya que en los últimos meses del gobierno de Martínez de Perón las relaciones quedaron paralizadas (Cisneros-Escudé, 2000).

En este contexto, los trabajos de Guglialmelli fijan una postura en torno al conflicto, empero, en función de su proyecto pragmático de desarrollo nacional. El mismo presenta una preponderancia del uso de concepciones geopolíticas, llegando al punto de funcionar como un eje articulador de sus producciones intelectuales ubicándolo, a su vez, dentro de la corriente nacional-territorialista (irrendentista). Así, uno de los postulados teóricos esgrimidos por la corriente nacional-territorialista (irrendentista) argentina es la denuncia de una política exterior equívoca, a la que Guglialmelli — con relación a la Sexta Reunión de Cancilleres de la Cuenca del Plata- consideró con escasa capacidad negociadora. Asimismo, en el mismo artículo, realiza una denuncia directa frente al aparente imperialismo de Brasil, apoyado desde los Estados Unidos:

“(...) Brasil, apoyado desde Washington, ha operado con objetivos claros, tenazmente perseguidos y mejor instrumentados, acicateado hoy, además. Por necesidades perentorias de materias primas y fuentes de energía. Avanza sobre los países menores; busca caminos hacia el Pacífico; coopera en el desarrollo de éstos en los sectores que su propia economía ha de requerir; bloquea la cooperación o proyectos de la Argentina; gana la derecha y construye grandes obras hidroeléctricas; presta ayuda técnica y financiera; abre, en fin, su litoral marítimo en Santos, Paranaguá y Rio Grande, para atraer hacia esos puertos, a través de caminos, ferrocarriles y canales al Oriente Boliviano, al Paraguay, al Uruguay y la Mesopotamia Argentina, en abierto reto y competencia al sistema portuario del Río de la Plata"22.

En este pasaje se evidencia el interés de Guglialmelli por enfrentar, lo que él considera, como un proyecto de desarrollo integral brasileño en múltiples frentes, para extender su dominio en detrimento de la Argentina. Esta denuncia de expansionismo, sin embargo, no se limita a este artículo, sino que es reiterado en otros trabajos ${ }^{23}$. La solución, frente a tal avance, no sería otra que el desarrollo integral y socio-económico de la nación, antes que el enfrentamiento directo entre ambos países. Además, tal proyecto de desarrollo, que nuestro autor interpreta como imperialista y de vocación hegemónica, se manifestaría en un Acuerdo Nuclear firmado entre Brasil y Alemania (Acuerdo de

${ }^{22}$ Guglialmelli, J. “¿Cuenca del Plata o Cono Sur? A propósito de la VI Reunión de Cancilleres de la Cuenca del Plata, Estrategia, n 28, mayo-junio de 1974, p. 14.

23 "Argentina- Brasil, enfrentamiento o alianza para la liberación". Estrategia $\mathrm{n}^{\circ}$ 36, septiembre-octubre de 1975, pp.12, 13 y 21; "Corpus-Itaipú. Tres batallas perdidas por la Argentina y, ahora peligrosas perspectivas: el papel de socio menor de Brasil", Estrategia, n 61-62, noviembre-diciembre de 1979, p. 551, en Guglialmelli (2007); “¿Cuenca del Plata o Cono Sur? A propósito de la VI Reunión de Cancilleres de la Cuenca del Plata", Estrategia, n²8, mayo-junio de 1974, p. 14; "Argentina- Brasil, enfrentamiento o alianza para la liberación". Estrategia $n^{\circ}$ 36, septiembre-octubre de 1975, p.13; "Geopolítica en la Argentina”, Estrategia, n46-47, mayo-junio, julio-agosto de 1977, p. 363, en Guglialmelli (2007). 
Di Renzo. Geopolítica, desarrollo económico y política exterior: Juan Enrique Guglialmelli y su proyecto de nación...

Cooperación Nuclear, firmado el día 27 de junio de 1975). El problema radicaría en que el país vecino decidiera emprender la fabricación de una bomba atómica, lo cual afectaría de manera sensible a la Seguridad nacional argentina. Ante tal situación, no propone que se inicie una carrera armamentista, sino que se debía llegar a un "acuerdo de información, consulta y eventual cooperación técnica en el campo nuclear que, entre otros aspectos determine seguridades efectivas y recíprocas ante la posible fabricación de artefactos nucleares, aunque éstos se destinen para fines pacíficos" ${ }^{24}$.

En otras palabras, Guglialmelli no considera que el enfrentamiento directo produzca resultados positivos para la Argentina y, en ocasiones, destaca que el balance de poder en Sudamérica se encuentra a favor de Brasil. El ideólogo del supuesto proyecto expansionista, por otro lado, no sería otro que el Barón de Río Branco ${ }^{25}$. A su vez, tal proyecto se encontraría sustentado por la doctrina geopolítica de los intelectuales militares Lysias Rodrigues y Golbery da Couto e Silva ${ }^{26}$.A diferencia del Brasil, en Argentina no se le prestaría la atención necesaria a tales doctrinas y se manifestaría en la subestimación de aspectos geopolíticos esenciales, tales como el componente espacial ${ }^{27}$. Para fundamentar esta afirmación, Guglialmelli recurre al mito virreinal, aquel que intelectuales del cambio de siglo XIX al XX, tales como Vicente Quesada, dan origen a través de sus escritos ${ }^{28}$. Al hacer referencia a este punto, Guglialmelli utiliza el concepto de "desmembramiento del Virreinato, lo cual nos remite a pensar en la consideración del espacio territorial tal como lo han hecho las corrientes geopolíticas europeas clásicas de mediados y finales del siglo XIX, principalmente a aquellos postulados provenientes de la Escuela de Múnich"29.

${ }^{24}$ Guglialmelli, J. “¿Y si Brasil fabrica la bomba atómica? A propósito del Acuerdo Nuclear BrasileñoAlemán, Estrategia n 34/35, mayo de 1976, pp. 223, En Guglialmelli (2007).

${ }^{25}$ José María da Silva Paranhos Junior, recibe el título de Barão do Rio Branco durante la última etapa del Imperio del Brasil. Estuvo a cargo del Ministerio de Relaciones Exteriores durante diez años (19021912), a lo largo de cuatro mandatos presidenciales. Fue a partir de la labor de Barão de Rio Branco que se emprendió una carrera armamentista con Argentina, justamente, por la influencia de la Doctrina Mahan sobre aquel funcionario en conjunto con las ideas desarrolladas por el geógrafo alemán F. Ratzel. Tal situación llevó a que se deterioraran las relaciones bilaterales entre estos países, puesto que, desde Argentina, Zeballos llevaba a cabo un discurso en términos similares, ya que para Rio Branco, las acciones realizadas por Brasil en relación con su política exterior estaban destinadas a establecer su supremacía en América del Sur (Heinsfeld, 2013).

${ }^{26}$ Lysias A. Rodrigues (1896-1957) fue un militar de carrera dentro de la Fuerza Aérea brasileña, especialista en geopolítica. Al respecto véase Meira Mattos, (1969) y Miyamoto (1981). Principal ideólogo de la dictadura militarbrasileña (1964-1984), Golbery do Couto e Silva desarrolló la estrategia de ocupación territorial del interior de Brasil, como tarea de seguridad para garantizar el control estatal sobre el territorio nacional basando en su doctrina geopolítica. Participó de varios gobiernos de la dictadura militar, que incluyen a Castelo Branco (1964-1966), Ernesto Geisel (1974-1979) y, finalmente, João Figueiredo (1979-1985). Al respecto véase Birkner (2002); Da Luz (2015) y Fernandes, (2009).

${ }^{27}$ Guglialmelli, J., "Argentina- Brasil, enfrentamiento o alianza para la liberación". Estrategia n ${ }^{\circ} 36$, septiembre-octubre de 1975, pp. 17.

${ }^{28}$ En el caso argentino, la producción intelectual de finales del siglo XIX, en las que se señalan las constantes pérdidas territoriales desde la época virreinal hacen surgir un "mito virreinal" (Cavaleri, 2012, p. 12).

${ }^{29}$ Guglialmelli, J., "Argentina-Brasil, enfrentamiento o alianza para la liberación". Estrategia $n^{\circ} 36$, septiembre-octubre de 1975, p 17. 


\section{El desarrollo económico y el rol de las Fuerzas Armadas}

Como hemos señalado, los planteos del general de división lo posicionan dentro del grupo de los nacionalistas-desarrollistas, quienes compartían los recelos de sus colegas "ortodoxos" por la "expansión" de Brasil y Chile. Pero incorporaron, además, nuevos elementos provenientes de argumentos entroncados con la teoría de la Dependencia y el desarrollismo, a las hipótesis de conflicto (Cisneros y Escudé, 2000). Así, percibieron en el contraste entre el "subdesarrollo" de las zonas fronterizas argentinas y el "desarrollo" chileno —o brasileño — un nuevo componente de amenaza. Convencidos de que la mejor forma de contener a estos vecinos expansionistas era a través de medidas de desarrollo e integración a nivel interno, los "desarrollistas" otorgaron especial énfasis a la necesidad de adoptar medidas de promoción y desarrollo económico en ciertas áreas descuidadas o subdesarrolladas como Misiones, el Chaco, o la Patagonia.

Por otra parte, el concepto de desarrollo en los trabajos de Guglialmelli, además de comprender el aspecto económico, es extensivo a otras áreas tales como la sociedad, la cultura y al espíritu de la nación, dando como resultado un concepto de desarrollo integral ${ }^{30}$. El énfasis en el fomento de la industria pesada, a su vez, lo acercan a los postulados de los desarrollistas sudamericanos, tales como lo de Rogelio Frigerio —en Argentina- y Helio Jaguaribe de Mattos en Brasil, entre otros ${ }^{31}$. Pues, precisamente, entre 1955 y 1970, la problemática del desarrollo atrajo e inspiró a una amplia franja intelectual (Altamirano, 1998, p. 79) y los artículos de la revista Estrategia dan cuenta de ello. De todas maneras, uno de los rasgos que diferencian el proyecto de desarrollo postulado por Guglialmelli, de otros teóricos contemporáneos, es el lugar que les asigna a las Fuerzas Armadas. Desde la aparición del primer número, en mayo-junio del año 1969, hasta el último de ellos, son 5 los artículos que tratan acerca del rol de las Fuerzas Armadas en la Argentina, al menos de manera directa ${ }^{32}$. No obstante, estos trabajos pertenecen a los primeros años de la revista y posteriormente no van a volver a tener recurrencia dentro del corpus total, siendo el último de ellos el que apareció en el $n^{\circ} 23$, de julio-agosto de 1973.Por consiguiente, existe una coincidencia entre esta problemática y los años en los que cumplió su rol como agente del gobierno de facto ya que, como hemos mencionado anteriormente, durante la gestión de Marcelo Levingston, fue nombrado en el cargo de Secretario del Consejo Nacional de Desarrollo (CONADE).

Ahora bien, ¿cuál debería ser el rol de las Fuerzas Armadas en su proyecto de nación? Por lo expuesto anteriormente, podemos sostener que compartía con los

\footnotetext{
${ }^{30}$ Guglialmelli, "Fuerzas armadas y subversión interior”, Estrategia, № 2, julio-agosto 1969, pp. 13-14. En lo que respecta a la asociación entre el desarrollo y el concepto de Seguridad, véase Snow (1972).

${ }^{31}$ Al respecto véase García Bossio (2008).

${ }^{32}$ Los artículos son: Guglialmelli, J., "Función de las Fuerzas Armadas en la actual etapa del proceso histórico argentino", Estrategia, n¹, mayo-junio de 1969, pp. 8-19; "Fuerzas armadas y subversión interior", Estrategia, No 2, julio-agosto 1969, pp. 7-14; Guglialmelli, J. "Responsabilidad de las Fuerzas Armadas en la Revolución Nacional", Estrategia, n4, noviembre-diciembre de 1969, pp. 7-17; "Las FF.AA. en América Latina (FF. AA. y Revolución Nacional)", Estrategia, n 17, julio-agosto de 1972, pp. 9-19; "Fuerzas Armadas para la Liberación Nacional”, Estrategia, n²3, julio-agosto de 1973, pp.130150, en Guglialmelli (2007).
} 
Di Renzo. Geopolítica, desarrollo económico y política exterior: Juan Enrique Guglialmelli y su proyecto de nación...

desarrollistas la idea de que la edificación de una estructura industrial integrada, así como el crecimiento económico general, debían ser deliberadamente promovidos (Altamirano, 1998, p. 80). Sus trabajos nos acercan al perfil de un militar de carrera, que se encontraba pensando de manera sistematizada en un proyecto para el desarrollo económico integral del país y, para ello, consideraba que las Fuerzas Armadas debían desempeñar un rol activo en la política nacional. Partiendo de la posibilidad de una hipótesis de conflicto armado (Chile y Brasil) y de la existencia de amenazas y agresiones en materia económica ejercidas por potencias extranjeras (Gran Bretaña, identificado como un agresor), sostiene que las Fuerzas Armadas deben encontrase a la vanguardia de la defensa de los intereses nacionales. Debían llevar a cabo un proyecto de integración territorial y económica, su proyecto, teniendo como uno de los objetivos el mantenimiento de la paz, ya sea a nivel interno (con relación a la denominada "subversión”), con los países vecinos, o bien en los aspectos ligados a la economía nacional ${ }^{33}$.

A nivel conceptual, el rol de las Fuerzas Armadas en un espacio atravesado por amenazas de orden interno y de orden externo, con una postergación en materia económica acumulada prácticamente desde los inicios de la Independencia (1816) —en un escenario internacional marcado por el mundo bipolar-, implicaba una verdadera revolución ${ }^{34}$. Nuestro autor utiliza esta noción (revolución) y la aúna al concepto de Nacional:

"Entendemos como tal esfuerzo orgánico de toda la comunidad para consolidar su rango de Nación, de manera que el centro de decisión soberana en todo aquello que resulte esencial, le pertenezca. Por lo expresado, constituyen objetivos inmediatos de esa Revolución construir las bases materiales de la soberanía y fortalecer los vínculos espirituales entre los sectores sociales y las distintas regiones por encima de sus distintas ideologías" 35 .

En este pasaje podemos evidenciar que el concepto de revolución aparece asociado al de desarrollo económico y, por ende, sin tal transformación, no sería posible la Defensa nacional y la integración local y regional a nivel latinoamericano. De esta manera, no había espacio para las dudas: el rol de las Fuerzas Armadas, en el proyecto de desarrollo e integración nacional, era central. Debido al impacto de doctrina, las autoridades de la Escuela Nacional de Guerra sostuvieron la necesidad de redefinir el concepto de seguridad, vinculándolo al de desarrollo (Gomes, 2017). Por otra parte, y como hemos anticipado, el posicionamiento de Guglialmelli con respecto a la Revolución

\footnotetext{
${ }^{33}$ Con relación al control interno y al rol de las Fuerzas Armadas, Guglialmelli apuesta al desarrollo económico como la forma más efectiva de seguridad. Llega a estas conclusiones a partir del análisis realizado por el Secretario de Defensa de los Estados Unidos, Robert Mc Namara, en el que se asocia lo conceptos de violencia y subdesarrollo. Al respecto véase Guglialmelli Fuerzas Armadas y subversión interior, $\mathrm{n}^{\circ} 2$ julioagosto 1969. Sobre la producción intelectual de Osiris Villegas, véase Di Renzo (2018).

${ }^{34}$ En materia de relaciones militares en el escenario bipolar, Guglialmelli se posiciona abiertamente bajo la esfera de los Estados Unidos de Norteamérica. Al respecto véase "Fuerzas Armadas para la Liberación Nacional", Estrategia, n²3, julio-agosto de 1973, p. 150. En Guglialmelli (2007).

${ }^{35}$ Guglialmelli, J. "Responsabilidad de las Fuerzas Armadas en la Revolución Nacional”, Estrategia, n4, noviembre-diciembre de 1969 , p.8.
} 
Argentina era más bien crítico. Señalaba, por ejemplo, en un artículo del año 1969, que la dirección nacional no estaría en el rumbo correcto, siendo necesario "replantear los objetivos del esfuerzo principal y los objetivos de los esfuerzos secundarios pero concurrentes al mismo fin" ${ }^{36}$. Aunque, tal vez, la crítica más directa — dentro de la revista - sea la realizada en el año 1972, al sostener que "Hoy, estoy persuadido que la Revolución Nacional ha fracasado en el logro de sus objetivos fundamentales"37. A partir de esta crítica, y de otras realizadas en artículos posteriores, podemos afirmar que este militar de carrera efectuaba críticas explicitas a los gobiernos de facto, sobre todo en materia de desarrollo económico. Tener un alto rango y prestigio dentro del Ejército Argentino, disponer de un órgano de difusión periódico (del cual era director) y de un proyecto de nación pragmático, le permitió a Guglialmelli realizar acusaciones, tanto a la dictadura de la Revolución Argentina como también a la del Proceso de Reorganización Nacional ${ }^{38}$. Sin duda, disponer de la revista Estrategia era de vital importancia para difusión de sus planteos. En este sentido, hemos señalado que una de las características de las revistas era la de apuntar a transformar el presente (Sarlo, 1992), y nuestro autor lo señala abiertamente al referirse a la educación e instrucción militar ${ }^{39}$.

Así, el rol de las Fuerzas Armadas no debía circunscribirse sólo al objetivo por el cual fueron creadas, sino que tendrían un papel central, de carácter revolucionario, en la transformación de la sociedad argentina, en la conducción política y en la organización de un proyecto de desarrollo económico integral. El mismo debía funcionar como el principal elemento de pacificación interna $\mathrm{y}$, además, como la única solución efectiva frente al aparente avance expansionista de los países vecinos como Brasil y Chile. Esto no equivaldría a decir que sólo a través del autoritarismo podría llevarse a cabo su proyecto de desarrollo, sino en reconocer en el actor militar las capacidades de ponerlo en marcha. De hecho, en el último número de la revista Estrategia, la redacción lo reconoce como un auténtico demócrata, convencido de que la construcción de la Nación sólo podía realizarse sobre un sólido basamento popular y con la activa participación del pueblo ${ }^{40}$.

\section{El conflicto Beagle: posición del general Guglialmelli}

En la citada Tesis de Maestría (Di Renzo, 2018) evidenciamos que, en el periodo comprendido entre 1977 — fecha de Recepción del Laudo Arbitral, rechazado

\footnotetext{
${ }^{36}$ Guglialmelli, J. "Responsabilidad de las Fuerzas Armadas en la Revolución Nacional”, Estrategia, n4, noviembre-diciembre de 1969, p. 16.

${ }^{37}$ Guglialmelli, J. "Las FF.AA. en América Latina (FF.AA. y Revolución Nacional)", Estrategia, n¹7, julio-agosto de 1972, p.18.

${ }^{38}$ Guglialmelli, J. "Una década de Estrategia”, Estrategia, n'55-56, noviembre-diciembre de 1978, enerofebrero de 1979, p.7; “Argentina. Geopolítica y fronteras. Estrategia n ${ }^{\circ}$ 57, marzo-abril de 1979, p.12; "El General Savio. Industrias básicas, poder militar y poder nacional”. Estrategia n ${ }^{\circ} 60$, septiembre-octubre de 1979, p.30; "Corpus-Itaipú. Tres batallas perdidas por la Argentina y, ahora peligrosas perspectivas: el papel de socio menor de Brasil", Estrategia, n 61-62, noviembre-diciembre de 1979, p. 553. En Guglialmelli (2007).

${ }^{39}$ Véase Guglialmelli, J., "Fuerzas Armadas para la Liberación Nacional”, Estrategia, n²3, julioagosto de 1973, p.148.

${ }^{40}$ Consejo de Redacción, "La muerte de nuestro fundador", Estrategia nº73, 1983, p.6.
} 
Di Renzo. Geopolítica, desarrollo económico y política exterior: Juan Enrique Guglialmelli y su proyecto de nación...

por el gobierno de facto argentino-y la firma del Acta de Montevideo en el año 1979, existieron expresiones de convivencia pacífica entre Argentina y Chile, tales como las reuniones entre representantes oficiales, con expresiones de violencia, tales como ejercicios de desembarco y de defensa hacia ambos lados de la frontera. A través del análisis de diversas fuentes, demostramos que se osciló entre una diplomacia parcial puesto que la representación exterior argentina no se encontraba unificada y confrontaban distintas posturas-, y entre la guerra total, ya que las tres Fuerzas Armadas Argentinas (Ejército, Armada y Fuerza Aérea) estaban en condiciones efectivas de dar inicio a las hostilidades. Asimismo, dejamos constancia de que la producción de especialistas civiles (académicos, políticos y/diplomáticos) y militares sobre la temática, tuvo un rol central en la construcción del conflicto, puesto que las tesis y los conceptos asociados a ellas, fueron utilizados como fundamento en el accionar de los distintos actores sociales involucrados, especialmente por aquellos que apostaban a la salida armada.

Por otra parte, hemos señalado que, en términos de códigos geopolíticos, Guglialmelli había distinguido cuáles eran los enemigos reales o potenciales y, en ese contexto, Chile aparecía como uno de ellos. Por ende, en los momentos de tensión entre ambos países, no resulta extraño que haya dedicado un documento especial denominado "Arbitraje sobre el Beagle" (abril-mayo de 1977), de carácter histórico y descriptivo, además de dos artículos propios: "El área meridional del Atlántico Suroccidental, la geopolítica de Chile y el laudo del Beagle, de septiembre-octubre de 1977 y "Cuestión Beagle. Negociación directa o diálogo de armas". Ambos correspondientes a los meses de noviembre-diciembre de 1977 y enero-febrero de 1978. En principio, debemos subrayar que al conocer el dictamen del laudo arbitral de 1977, nuestro autor se encumbra bajo el sector que rechaza al mismo, esgrimiendo que la Corte Arbitral actuó con un evidente "exceso de poder" ${ }^{41}$. Asimismo, y señalando la importancia geopolítica del área en disputa (el canal Beagle y las islas Picton, Lennox y Nueva), no duda en calificar de expansionista la postura chilena, la que estaría aprovechando el "descuidismo fronterizo" argentino (equívoca conducción de la Política Exterior) para romper con su "encierro geopolítico", acusación que se reitera en otros trabajos ${ }^{42}$. No obstante, en el primer artículo señala la necesidad de fomentar una política en común entre Argentina y

\footnotetext{
${ }^{41}$ Guglialmelli, J., "El área meridional del Atlántico Suroccidental, la geopolítica de Chile y el laudo del Beagle", Estrategia, n48, septiembre-octubre de 1977, p.5. El Dictamen de la Corte Arbitral mostró diferentes posiciones que pueden interpretarse como "dura, intermedia y benévola" (Lanús, 2000, p. 517; Russell, 1990, p. 47). La primera posición, sostenía que se debía rechazar el Laudo y estaba representada por una parte del Ejército y la Armada. Una segunda posición sostenía rechazar los "considerandos" y aceptar la "parte dispositiva", esgrimida por parte de la Cancillería. Por último, existía un sector ligada a la Consejería Legal y parte de la Fuerza Aérea que consideraba la aceptación del Laudo.

${ }^{42}$ En varias ocasiones, Guglialmelli resalta el aparente expansionismo chileno sobre el territorio argentino. Véase, Guglialmelli, J., "El área meridional del Atlántico Suroccidental, la geopolítica de Chile y el laudo del Beagle, Estrategia n $^{\circ}$ 48, septiembre-octubre de 1977, pp. 12-13; "Los intereses vinculados al statu quo postergan la integración patagónica", Estrategia, n ${ }^{\circ}$, septiembre-octubre de 1969, p.15; "Cuestión Beagle. Negociación directa o diálogo de armas", Estrategia n49-50, noviembre-diciembre de 1977, enero-febrero de 1978, p.397, en Guglialmelli (2007); "Argentina. Geopolítica y fronteras. Estrategia n $^{\circ}$ 57, marzo-abril de 1979, pp. 7-8; "Patagonia. A cien años de su ocupación no podemos conmemorar su vertebración a la nación”, Estrategia, n59, julio-agosto de 1979, p.16.
} 
Chile, tendiente a enfrentar los intereses de países ajenos a la región, para lo cual recurre a los elementos que los unen, tales como el idioma, la geografía y la historia ${ }^{43}$.

A medida que pasaban los meses, y los gobiernos de Argentina y Chile no lograban destrabar el conflicto por medio de la vía diplomática, la posibilidad de la guerra aparecía como una alternativa para un sector de las Fuerzas Armadas. De hecho, el Operativo Soberanía en Argentina y los planes de defensa chilenos dan cuenta de ello ${ }^{44}$. Guglialmelli era consciente de esta situación, y si bien sus postulados apostaban a salidas pacíficas de los conflictos existentes, no descartaba la opción por las armas. Así, en el segundo artículo referido al conflicto Beagle, se señala tal posibilidad:

“(...) Las fronteras, en particular patagónica, con muy serias vulnerabilidades. Hay, por lo tanto, requerimientos insoslayables que hacen al desarrollo integral de nuestra sociedad y al específico del conflicto bélico posible (...) Esta hipótesis bélica, no buscada pero presente en el horizonte, debe pesar en el ánimo de los negociadores como un estímulo para encontrar soluciones.

(...)Y si los hechos volvieran a repetirse, si persiste la tozudez chilena, a la instancia agotada, seguirá una única y trágica opción: imponer el derecho mediante el diálogo de armas. Pero conviene insistir. No será culpa argentina" ${ }^{45}$.

En otras palabras, la posición de Guglialmelli, cercana al sector que apostaba a una salida pacífica del conflicto, a medida que no se encontraban soluciones por los medios diplomáticos, consideró la opción por las armas como uno de los mecanismos de resolución de la disputa limítrofe. No obstante, esto no quiere decir que presenta una postura belicosa tal como otros exponentes de la corriente nacional-territorialista (irrendentista), sino que, como hombre de armas, la guerra es una posibilidad real para la que son preparados a lo largo de su carrera. Una y otra vez, la única solución real ante este conflicto y frente a los otros que señala, es el desarrollo económico integral de la nación. Sólo de esta manera las negociaciones con los países vecinos podrán adquirir otro carácter, siendo ésta la mejor forma de defensa. Pasemos entonces a realizar algunos balances generales.

\section{A modo de conclusión}

En las décadas de 1960, 1970 e inicios de 1980, la intelectualidad militar estuvo particularmente activa. Año tras año, sus trabajos aparecían en artículos, libros, conferencias y otras formas de divulgación tales como notas en periódicos. En este sentido, las diferencias existentes entre Argentina, Brasil y Chile en torno a

\footnotetext{
${ }^{43}$ Guglialmelli, J., "El área meridional del Atlántico Suroccidental, la geopolítica de Chile y el laudo del Beagle, Estrategia n $^{\circ}$ 48, septiembre-octubre de 1977, p.18.

${ }^{44} \mathrm{Al}$ respecto véase Madrid Murúa (2003) y Passarelli (1998).

${ }^{45}$ Guglialmelli, J., "Cuestión Beagle. Negociación directa o diálogo de armas", Estrategia n49-50, noviembre-diciembre de 1977, enero-febrero de 1978, p.400, en Guglialmelli, 2007.
} 
Di Renzo. Geopolítica, desarrollo económico y política exterior: Juan Enrique Guglialmelli y su proyecto de nación...

la delimitación fronteriza o en aspectos ligados al aprovechamiento de los recursos naturales compartidos, proporcionaron la base sobre la cual se desplegaron un gran corpus de escritos en los que las concepciones geopolíticas aparecen con fuerza. Pues en mayor o menos medida, la utilización de determinados códigos geopolíticos entre integrantes de las Fuerzas Armadas argentinas, dedicados al análisis de estos problemas, era recurrente y en el caso de Guglialmelli, son el eje vertebrador de todos sus artículos.

No obstante, si bien existían ciertas equivalencias a la hora de señalar el origen y los responsables de la situación de "crisis" que les tocaba transitar, no todos los integrantes de la corriente nacional-territorialista irrendentista planteaban soluciones. Algunos simplemente se detenían en las denuncias de la situación, que reconocían como injusta y producto del devenir histórico poco favorable para la Argentina, sobre todo, en materia de delimitación fronteriza. Otros, civiles o militares, fortalecían la imagen negativa sobre Chile o Brasil, al representarlos como países expansionistas que atentan contra la integridad territorial heredada desde la etapa virreinal. Estas ideas eran compartidas por Guglialmelli, empero, sus análisis por lo general apuntaban más a las soluciones que a los problemas. En este sentido, el aporte del general de división radica en la elaboración de una teoría pragmática de desarrollo integral que comprende múltiples áreas, pero que se basa, en principio, en la esfera económica. Desde allí se solucionarían distintos problemas tales como las disputas territoriales y los déficits existentes en Seguridad y Defensa. Por ende, el énfasis se encontraba en el desarrollo de sectores considerados estratégicos para el desarrollo, tales como la energía, la minería, la siderurgia y la química pesada.

En suma, en este trabajo pretendimos contribuir a la vacancia existente dentro de la historiografía sobre la intelectualidad militar en el campo de la geopolítica. A lo largo de los 14 años que tuvo aparición la revista Estrategia, con sus 74 números, Guglialmelli fue forjando una doctrina que lo posiciona como uno de los referentes dentro de la escuela argentina y, más aun, dentro del Ejército.

\section{Referencias Bibliográficas}

Agnew, J. (2005). Geopolitica: una re-visión de la política mundial. Trama editorial, Madrid. Altamirano, C. (1998). "Desarrollo y desarrollistas". Prismas. Revista de historia intelectual, no 2, p. 75-94. Avellaneda, A. (2019). "La primera guerra mundial y la conducta económica del Estado en el pensamiento militar en Argentina". Iberian Journal of the History of Economic Thought, $\mathrm{n}^{\circ}$ 6, pp. 17-35.

Cairo, H. (2012). "La geopolítica como "ciencia de Estado": el mundo del general Haushofer". Geopolitica (s). Revista de estudios sobre espacio y poder, no 2, p. 337-345.

Cavaleri, P. (2004). La restauración del Virreinato. Orígenes del nacionalismo territorial argentino. Universidad Nacional de Quilmes, Bernal.

Cornut, H. (2018). "Los militares, la industria y el petróleo. Pensar la guerra y la Nación (1923-1930). En L. Rodríguez y G. Soprano, (ed.) Profesionales e intelectuales de Estado. Análisis de perfiles y trayectorias en la salud pública, la educación y las fuerzas armadas. Prohistoria, Rosario. 
Cuéllar, R. (2012). “Geopolítica. Origen del concepto y su evolución”. Revista de Relaciones Internacionales de La UNAM, no 113, pp. 59-80.

Da Luz, A. (2015). "Geopolítica do Brasil: a trajetória de Golbery do Couto e Silva e sua perspectiva no campo intelectual". Revista Ágora, no 22, p. 350-360.

Cisneros, A. y Escudé, C. (1998). Historia general de las relaciones exteriores de la República Argentina, Tomo XIV, cap. 66 y 67. Centro de Estudios de Política Exterior (CEPE) Consejo Argentino para las Relaciones Internacionales, Buenos Aires.

Deciancio, M. (2017). "La construcción del campo de las Relaciones Internacionales argentinas: contribuciones desde la geopolítica". Revista de Relaciones Internacionales, Estrategia y Seguridad, N¹2, pp.179-205.

Di Renzo, C. (2018). Entre la Diplomacia parcial y la guerra total. Concepciones geopolíticas de militares argentinos en el marco del conflicto por el canal de Beagle y las islas Picton, Lennox y Nueva (1977-1979). Tesis de Maestría en Historia por la UNMDP, Mar del Plata.

Di Renzo, C. (en prensa). "Concepciones geopolíticas en torno a la política regional de argentina: La Revista Argentina de Relaciones Internacionales. 1975-1981". Coordenadas. Revista de Historia Local y Regional. Aprobado para su publicación.

Escudé, C. (2008). "Apuntes sobre los orígenes del nacionalismo territorial argentino". Serie Documentos de Trabajo. Buenos Aires: Universidad del CEMA. Área: ciencia política, $\mathrm{n}^{\circ} 388$.

Fajardo, J. (2004). Acordo Tripartite Itaipu - Corpus: ponto de inflexão entre a disputa geopolítica e a política de cooperação. Tesis de Maestría em Ciencias Políticas, Universidade Federal do Rio Grande do Sul.

Fernandes, A. (2009). "A reformulação da Doutrina de Segurança Nacional pela Escola Superior de Guerra no Brasil: a geopolítica de Golbery do Couto e Silva". Antiteses, 2009, no 4, p. 831-856.

Fernandes, M. (2013). "El conocimiento geopolítico de José Marini e a geopolítica clássica revisitada: o conceito de geopolítica, o seu objeto e método de estudo". Nação e Defesa, n¹36, p. 277-299.

Flint, C. (2006). Introduction to Geopolitics. Routledge, Oxford.

Fornillo, B. (2015). "Centralidad y permanencia del pensamiento geopolítico en la historia reciente de Sudamérica” (1944-2015).Estudios Sociales del Estado, № 2, pp. 118-148.

García, H. (2008). "Génesis del estado desarrollista latinoamericano: el pensamiento y la praxis política de Helio Jaguaribe (Brasil) y de Rogelio Frigerio (Argentina)". Documento de trabajo No. 23 del Departamento de Economía de la Facultad de Ciencias Sociales y Económicas de la Universidad Católica Argentina), Buenos Aires.

Gomes, G. (2017). "Baires 2000. Imaginarios y visiones de futuro en las agencias de Planeamiento y Desarrollo de las dictaduras militares de Argentina (19661983)". Estudios Sociales del Estado, no 6, p. 66-93.

Heinsfeld, A. (2013). “A influencia de Ratzel e Mahan na política externa do Barão do Rio Branco". Revista de Geopolítica, no 1, p. 115-134.

Kosselleck, R. (1992). Futuro pasado: para una semántica de los tiempos históricos. Paidós, Buenos Aires. 
Di Renzo. Geopolítica, desarrollo económico y política exterior: Juan Enrique Guglialmelli y su proyecto de nación...

Laguado Duca, A. (2010). “Cuestión social, desarrollo y hegemonía en la Argentina de los años sesenta. El caso de Onganía”. Universitas humanística, no 70, p. 101-118.

Lanús, J. (2000). De Chapultepec al Beagle. Política exterior argentina: 1945-1980. Emecé, Buenos Aires.

Larra, R. (1995). La batalla del general Guglialmelli. Distal, Buenos Aires.

Leal Buitrago, F. (2003). "La doctrina de Seguridad Nacional: materialización de la Guerra Fría en América del Sur”. Revista de estudios sociales, no 15, p. 74-87.

Madrid Murúa, R. (2003). "La Estrategia Nacional y Militar que planificó Argentina, en el marco de una estrategia total, para enfrentar el conflicto con Chile el año 1978”. Memorial Del Ejército de Chile, nº 471, p. 50-69.

Meira Mattos, C. (1969). “A geopolítica brasileira-predecessores e geopolíticos”. Revista da Escola Superior de Guerra, no 39, p. 58-82.

Mendoza Pinto, J. (2017). Razonamiento geopolítico. Construcción de representaciones y códigos geopolíticos de Chile y sus vecinos. Editorial Universidad de Concepción, Concepción.

Miyamoto, S. (1981.) “Os estudos geopolíticos no Brasil: uma contribuição para sua avaliação”. Perspectivas: Revista de Ciências Sociais, 1981, no 1, p. 75-92.

O’Donnell, G. (1981) El Estado burocrático-autoritario. Editorial de Belgrano, Buenos Aires.

Pécora, G. (2013). "De leales a conjurados. Una expresión de la derecha nacionalistacatólica de 1955 en Río Cuarto". Historia Regional, no 31, p. 79-88.

Palti, E. (2007). La nueva historia intelectual y sus repercusiones en América Latina. Revista de Historia Unisinos, sept.-dic. p. 297-305.

Passarelli, B. (1998). El delirio armado. Argentina-Chile. La guerra que evito el Papa. Sudamericana, Buenos Aires.

Potash, R. (1982). El ejército y la politica en la Argentina: 1945-1962 de Perón a Frondizi. Sudamericana, Buenos Aires.

Russell, R. (1990). Politica exterior y toma de decisiones en América Latina. Grupo Editor Latinoamericano, Buenos Aires.

Sarlo, B. (1992). "Intelectuales y revistas: razones de una práctica". América. Cahiers du criccal, no 1, p. 9-16.

Segre, M. (1990). La cuestión Itaipú-Corpus: el punto de inflexión en las relaciones argentino-brasileñas.Serie de Documentos e Informes de investigación, No 97 , FLACSO, Buenos Aires.

Snow, P. (1972). "Desarrollo económico y seguridad nacional en el régimen militar argentino". Estudios Internacionales, no 20, p. 67-74.

Velásquez Rivera É. (2002). "Historia de la Doctrina de la Seguridad Nacional". Convergencia Revista de Ciencias Sociales, no 27, p. 11-39.

Quintero, S. (2004). "Los textos de Geografía: un territorio para la nación”, en Romero, L. (coord.). La Argentina en la escuela. La idea de nación en los textos escolares. Siglo XXI, Buenos Aires. 


\section{Fuentes}

Guglialmelli, J., "Propósitos y definiciones". Estrategia n¹, mayo-junio, 1969, pp. 5-7. Guglialmelli, J., "Función de las Fuerzas Armadas en la actual etapa del proceso histórico argentino", Estrategia, n ${ }^{\circ}$, mayo-junio de 1969, pp. 8-19.

Guglialmelli, J., "Fuerzas armadas y subversión interior", Estrategia, № 2, julioagosto 1969 , pp. 7-14.

Guglialmelli, J., "Los intereses vinculados al statu quo postergan la integración patagónica", Estrategia, n³, septiembre-octubre de 1969, pp. 8-23.

Guglialmelli, J., "Responsabilidad de las Fuerzas Armadas en la Revolución Nacional", Estrategia, $\mathrm{n}^{\circ}$ 4, noviembre-diciembre de 1969, pp. 7-17.

Guglialmelli, J., 120 días en el gobierno. S/r, Buenos Aires.

Guglialmelli, J., "Las FF. AA. en América Latina (FF.AA. y Revolución Nacional)", Estrategia, ${ }^{\circ} 17$, julio-agosto de 1972, pp.9-19.

Guglialmelli, J., "Fuerzas Armadas para la Liberación Nacional", Estrategia, n²3, julio-agosto de 1973, pp.130-150, en Guglialmelli (2007).

Guglialmelli, J., “¿Cuenca del Plata o Cono Sur? A propósito de la VI Reunión de Cancilleres de la Cuenca del Plata, Estrategia, n 28, mayo-junio de 1974, pp. 7-17.

Guglialmelli, J., "¿Y si Brasil fabrica la bomba atómica? A propósito del Acuerdo Nuclear Brasileño-Alemán”, Estrategia n 34/35, mayo de 1976, pp. 215-223, En Guglialmelli (2007).

Guglialmelli, J., "Argentina- Brasil, enfrentamiento o alianza para la liberación". Estrategia n ${ }^{\circ}$ 36, septiembre-octubre de 1975, pp. 1-29.

Guglialmelli, J., "Argentina, Política nacional y problemas de fronteras. Crisis nacional y problemas fronterizos". Estrategia ${ }^{\circ} 37 / 38$, noviembre-diciembre de 1975 y enero y febrero de 1976, pp. 5-21.

Guglialmelli, J., ¿Argentina insular o peninsular? Reflexiones geopolíticas en torno al pensamiento del Vicealmirante Segundo R. Storni”. Estrategia $n^{\circ}$ 40/41, mayojunio, julio-agosto de 1976, pp. 5-25.

Guglialmelli, J., "Geopolítica en la Argentina”, Estrategia, n 46-47, mayo-junio, julioagosto de 1977, pp. 360-369, en Guglialmelli (2007).

Guglialmelli, J., "El área meridional del Atlántico Suroccidental, la geopolítica de Chile y el laudo del Beagle", Estrategia, nº48, septiembre-octubre de 1977, pp. 5-18.

Guglialmelli, J., "Cuestión Beagle. Negociación directa o diálogo de armas", Estrategia $\mathrm{n}^{\circ} 49-50$, noviembre-diciembre de 1977, enero-febrero de 1978, pp.384-401, en Guglialmelli (2007).

Guglialmelli, J., "Economía, Poder Militar y Seguridad Nacional". Estrategia n 51, marzo-abril de 1978, pp. 7-29.

Guglialmelli, J., "Una década de Estrategia, Estrategia, n55-56, noviembre-diciembre de 1978, enero-febrero de 1979, pp.5-8.

Guglialmelli, J., "Argentina. Geopolítica y fronteras. Estrategia ${ }^{\circ}$ 57, marzo-abril de 1979 , pp. 5-15.

Guglialmelli, J., "Patagonia. A cien años de su ocupación no podemos conmemorar su vertebración a la nación”, Estrategia, n59, julio-agosto de 1979, pp.5-36. 
Di Renzo. Geopolítica, desarrollo económico y política exterior: Juan Enrique Guglialmelli y su proyecto de nación...

Guglialmelli, J., "El General Savio. Industrias básicas, poder militar y poder nacional". Estrategia $\mathrm{n}^{\circ} 60$, septiembre-octubre de 1979, pp.5-36.

Guglialmelli, J., "Corpus-Itaipú. Tres batallas perdidas por la Argentina y, ahora peligrosas perspectivas: el papel de socio menor de Brasil", Estrategia, ${ }^{\circ}$ 61-62, noviembre-diciembre de 1979, pp.538-556, en Guglialmelli (2007).

Guglialmelli, J., Consejo de Redacción, "La muerte de nuestro fundador. In Memoriam", Estrategia $\mathrm{n}^{\circ} 73,1983$, pp.5-8. 